\title{
PLANE GEOMETRIES IN TERMS OF PROJECTIONS ${ }^{1}$
}

\section{HOWARD LEVI}

In Artin [1, pp. 51-75] the affine plane is developed and coordinatized from a system of four axioms. The present paper also develops affine plane geometry from a system of four axioms. The first three are the same as Artin's. The fourth is concerned with parallel projections from lines to lines. It leads to a construction of a skew field in a fashion analogous to Bachmann's [2, pp. 137-140] construction of a commutative field. This field can then be used to coordinatize the plane as in Levi [3, pp. 32-37], [4, pp. 50-53]. Both Artin's treatment and the present one deal with mappings rather than configurations. They differ in that Artin considers mappings of the whole plane into itself, whereas here we deal only with mappings from individual lines to lines. Each of our axioms has a natural counterpart for projective geometry, and our development of affine geometry suggests a parallel development of projective geometry. However, the field which this approach yields is necessarily commutative. To cover the general case we present a system of four axioms for the projective plane, three being counterparts as stated, and the fourth a modified counterpart. This fourth axiom, which is a weakened form of the "Fundamental Theorem of Projective Geometry," can also be regarded as a form of Desargues' Theorem, in which only those instances are asserted which contain a prescribed line in a prescribed place in the configuration. The author wishes to thank H. S. M. Coxeter and Joseph Lipman for comments on an earlier version of this paper which resulted in substantial simplifications.

Terminology and the first three axioms. We suppose that $Q$ is a set, and that $\mathscr{L}$ is a set of subsets of $a$. We call the elements of $a$ points and the elements of $\&$ lines. We say that lines $l$ and $l^{\prime}$ are parallel if and only if either $l=l^{\prime}$ or they have no point in common.

Axıom I. If $A$ and $B$ are distinct points there is one and only one line which contains them.

Axrom II. If $L$ is any point and if $l$ is any line there is one and only one line which contains $L$ and is parallel to $l$.

It follows directly from these axioms that parallelism is an equivalence relation on $\mathcal{L}$, and that two nonparallel lines have exactly one

Received by the editors September 17, 1963 and, in revised form, January 10, 1964.

1 This work was supported in part by the National Science Foundation under grant GP1231. 
point in common. If $l$ and $l^{\prime}$ are lines and if $m$ is a line parallel to neither, then parallel projection from $l$ to $l^{\prime}$ in the direction of $m$ is the function whose value at each point $X$ of $l$ is the intersection with $l^{\prime}$ of the line through $X$ parallel to $m$. It follows directly from these axioms that parallel projection from $l$ to $l^{\prime}$ is a one-to-one correspondence from $l$ to $l^{\prime}$.

Axiom III. There are three noncollinear points.

It follows from these axioms that every point is contained in at least three lines, that every line contains at least two points, and that all lines have the same cardinality.

We use the notations $A B$ to denote the line which contains the distinct points $A$ and $B$, and $\|$ to denote parallelism.

Affinities. We say that a function is an affinity if and only if it is a finite composition of parallel projections.

THEOREM 1. Let $l$ be any line and let $G(l)$ be the set of all affinities from $l$ to itself. Then $\mathrm{g}(l)$ is a group, relative to composition. If $l$ and $l^{\prime}$ are any lines, and if $f$ is any affinity from $l$ to $l^{\prime}$, then the mapping from $\mathcal{G}(l)$ to $G\left(l^{\prime}\right)$ whose value at $x$ is $f x f^{-1}$ is an isomorphism.

Proof. Since parallel projections have inverses which are parallel projections, affinities have inverses which are affinities, and, in particular, the inverses of elements of $g(l)$ also belong to $g(l)$. It is immediate that $\mathcal{G}(l)$ has the other group properties. That $x \rightarrow f x f^{-1}$ is a homomorphism follows from the fact that $\left(f x f^{-1}\right)\left(f y f^{-1}\right)=f x y f^{-1}$. That it is one-to-one and onto follows from the fact that it has an inverse whose value at each $z$ of $g\left(l^{\prime}\right)$ is $f^{-1} z f$.

Theorem 2. If $A, B, A^{\prime}, B^{\prime}$ are any points for which $A \neq B, A^{\prime} \neq B^{\prime}$, then there is an affinity $f$ for which $f(A)=A^{\prime}$ and $f(B)=B^{\prime}$.

Proof. We construct such an affinity for the case in which the given points are collinear. The general case is handled by performing any parallel projection from $A B$ to $A^{\prime} B^{\prime}$ and then composing it with the affinity devised for the collinear case.

If $A=A^{\prime}$ and $B=B^{\prime}$, then the identity affinity on $A B$ meets the requirements. Suppose now that $A \neq A^{\prime}$. Let $P$ be any point not on $A B$. Then $A P \nmid \mid A^{\prime} P$ and the line through $B$ parallel to $A P$ is not parallel to the line through $B$ parallel to $A^{\prime} P$. Denote their intersection point by $Q$. We assert that $Q \neq P, P Q \nmid \mid A P$ and $P Q \nmid \mid A^{\prime} P$. These are consequences, respectively, of the facts that $Q$ is on a line through $B$ parallel to $A P$ and $B$ is not on $A P$, that $P, Q, A$ are not collinear and that $P, Q, A^{\prime}$ are not collinear. Then the parallel projection from $A B$ to $P Q$ in the direction of $A P$ followed by the parallel projection from 
$P Q$ to $A B$ in the direction of $P A^{\prime}$ is an affinity which sends $A$ to $A^{\prime}$ and $B$ to $B^{\prime}$.

Our final axiom is the affine counterpart of the "Fundamental Theorem of Projective Geometry." A portion of Desargues' Theorem is readily deducible from it, and the subsequent development reveals that it implies the entire theorem and its converse.

Axıом IV. If $A$ and $B$ are distinct points and if $f$ and $g$ are affinities such that $f(A)=g(A)$ and $f(B)=g(B)$ then $f=g$.

This axiom implies immediately that the affinity of Theorem 2 is unique and that $\mathcal{G}(l)$ is sharply doubly transitive.

Some subgroups of $g(l)$. If $l$ is any line and if 0 is any point of $l$, it is clear that the set of all affinities from $l$ to $l$ having 0 as fixed point is a subgroup of $\mathcal{G}(l)$. We denote it by $\mathfrak{T l}_{0}(l)$. It is also clear that if $f$ is an affinity from $l$ to some line $l^{\prime}$ then the isomorphism from $\mathcal{G}(l)$ to $\mathcal{G}\left(l^{\prime}\right)$ it induces, restricted to $\mathfrak{T}_{0}(l)$, is an isomorphism from $\mathfrak{T}_{0}(l)$ to $\mathfrak{T}_{f(0)}\left(l^{\prime}\right)$. The following theorems identify another subgroup of $\mathcal{G}(l)$.

TheOREM 3. If $A$ and $A^{\prime}$ are distinct points there is one and only one affinity $f$ from $A A^{\prime}$ to itself which has no fixed points and for which $f(A)=A^{\prime}$.

Proof. Let $P$ be a point not on $A A^{\prime}$ and let $m$ be the line through $P$ parallel to $A A^{\prime}$. Then parallel projection from $A A^{\prime}$ to $m$ in the direction of $A P$ followed by parallel projection from $m$ to $A A^{\prime}$ in the direction of $P A^{\prime}$ is a fixed-point free affinity $f$ from $A A^{\prime}$ to itself for which $f(A)=A^{\prime}$. Suppose $f^{\prime}$ is a different affinity from $A A^{\prime}$ to itself for which $f^{\prime}(A)=A^{\prime}$. We show that $f^{\prime}$ has a fixed point. Let $B$ be a point for which $f(B) \neq f^{\prime}(B)$. According to our construction there is a point $Q$ on $m$ such that $A P \| B Q$ and $P A^{\prime} \| Q f(B)$. Since $f(B) \neq f^{\prime}(B)$, the point $Q^{\prime}$, where $B Q$ intersects the line through $f^{\prime}(B)$ parallel to $P A^{\prime}$, is different from $Q$, and, since it is not on $m$, it is different from $P$. Therefore $P Q^{\prime} \nmid \mid m$ and so $P Q^{\prime} \nmid \mid A A^{\prime}$. Let 0 be the intersection of $P Q^{\prime}$ with $A A^{\prime}$. Then the parallel projection from $A A^{\prime}$ to $P Q^{\prime}$ in the direction of $A P$ followed by the parallel projection from $P Q^{\prime}$ to $A A^{\prime}$ in the direction of $P A^{\prime}$ is an affinity from $A A^{\prime}$ to itself which agrees with $f^{\prime}$ on the distinct points $A$ and $B$. It follows that this affinity is $f^{\prime}$, and, since each of these parallel projections leaves 0 fixed, so does $f^{\prime}$.

LEMmA. If $A$ and $B$ are distinct points and if $f$ is an affinity for which $f(A)=B$ and $f(B)=A$ then, for every $X$ of $A B$, we have $f^{2}(X)=X$.

Proof. Since $f$ has the same value as $f^{-1}$ at $A$ and $B$, Axiom IV implies that $f=f^{-1}$. 
THEOREM 4. Let $B(l)$ be the set of all fixed-point free elements of $\mathcal{G}(l)$, together with the identity. Then $B(l)$ is an invariant abelian subgroup of $\mathcal{G}(l)$.

Proof. We show that if $t$ and $s$ are elements of $B(l)$ then so is $t s$. If $t s$ has no fixed point this is immediate. If $t s$ has the fixed point $X$ then we have $t(s(X))=s^{-1}(s(X))$. Theorem 3 implies that $t=s^{-1}$ so that $t s$ is the identity of $B(l)$.

Let $f$ be any element of $\mathcal{G}(l)$ and let $t$ be any element of $B(l)$. If $f t f^{-1}$ has the fixed point $X$ then $t$ has the fixed point $f^{-1}(X)$ and is the identity. Thus $f \otimes(l) f^{-1}$ is contained in $B(l)$.

There remains to show that $t s=s t$. If any one of $t, s$, or $s t$ is the identity then this is evident. If not let $A$ be any point of $l$. Then $t(A)$, $s(A)$ and $s t(A)$ are all different from $A$. Let $S$ be any point not on $l$, let $c$ be the line through $S$ parallel to $l$, let $T$ be the intersection of $c$ with the line through $t(A)$ parallel to $A S$. Then the parallel projection from $l$ to $c$ in the direction of $A S$ followed by the parallel projection from $c$ to $l$ in the direction of $S s(A)$ is an affinity of $B(l)$ which sends $A$ to $s(A)$. Since it must, therefore, be the same as $s$, it sends $t(A)$ to $s t(A)$, whence $T t(A) \| A S$. Consider now the parallel projections $f, g, h$, from $l$ to $T s t(A)$ to $A S$ to $l$ in the respective directions of $A S, l, T s t(A)$. The respective images of $A, t(A), s t(A)$ under $h g f$ are $s t(A), s(A), A$. Since $h g f$ interchanges $A$ and $s t(A)$ it follows from our lemma that $h g f(s(A))=t(A)$. It follows then that the parallel projection from $l$ to $f(s(A)) g f(s(A))$ in the direction of $A S$ followed by the parallel projection back to $l$ in the direction of $T s t(A)$ is a fixed-point free affinity from $l$ to itself which sends $A$ to $t(A)$ and $s(A)$ to $\operatorname{st}(A)$. The first of these facts implies that this affinity is $t$. We infer that $t s(A)=s t(A)$, whence $t s=s t$.

CoROLlaRy. If $f$ is an affinity from $l$ to $l^{\prime}$, then the isomorphism from $\mathcal{G}(l)$ to $\mathcal{G}(l)$ it induces, restricted to $B(l)$, is an isomorphism from $B(l)$ to $B\left(l^{\prime}\right)$.

Construction of the field. Let $l$ be any line and let 0 and $I$ be any distinct points of $l$. For each $X$ and $Y$ of $l$ let $B_{X}^{Y}$ be the element of $B(l)$ which sends $X$ to $Y$, and for each $X$ and $Y$ different from 0 let $M_{X}^{Y}$ be the element of $\Re_{0}(l)$ which sends $X$ to $Y$. For every $X$ and $Y$ of $l$ we define $X+Y$ to be $B_{0}^{X}(Y)$, we define $X \cdot Y$ to be $M_{I}^{X}(Y)$ if $X \neq 0$ and we define $X \cdot Y$ to be 0 if $X=0$. Then, if we identify our $B(l)$ with Bachmann's $T$ [2, pp. 137-140], our $\mathfrak{N}_{0}(l)$ with his $D$ and our $l$ with his $K$, his arguments apply. They show that, relative to + , the line $l$ is an abelian group whose identity element is 0 and whose 
negatives are given by $-X=B_{X}^{0}(0)$, that, relative to $\cdot$, the set $l-\{0\}$ is a group whose identity element is $I$, whose inverses are given by $X^{-1}=M_{X}^{I}(I)$ and that for every $X, Y$ and $Z$ of $l$ we have $X \cdot(Y+Z)=(X \cdot Y)+(X \cdot Z)$. Although he did not invoke this fact in his proofs, Bachmann assumed that $D$ was commutative, and so was able to conclude from these facts that his $K$ was a field. Since our $\mathfrak{T}_{0}(l)$ is not necessarily commutative we must establish that $(Y+Z)$ $\cdot X=(Y \cdot X)+(Z \cdot X)$ in order to conclude that $l$ is a skew field. We do so now.

THEOREM 5. Let $A$ be any point of $l$ other than $-I$. Then the function from $l$ to $l$ given by $X \rightarrow(A \cdot X)+X$ is the affinity $M_{I}^{A+I}$ and $(A \cdot X)+X$ $=(A+I) \cdot X$.

Proof. If $A$ or $X$ is 0 the result is immediate. Otherwise let $m$ and $n$ be lines through 0 distinct from $l$ and from one another, let $f_{l m}$ be the parallel projection from $l$ to $m$ in the direction of $n$, and let $f_{m n}$ be the parallel projection from $m$ to $n$ in the direction of $l$. Let $P$ be $f_{m n} f_{l m}(I)$. Since $A$ is neither 0 nor $-I$ the line $A P$ is parallel neither to $m$ nor to $n$. Let $f_{m l}$ and $f_{n l}$ be the respective parallel projections from $m$ and $n$ to $l$ in this direction. We evaluate $f_{l m} f_{m l}(X)$ in two ways. We can regard this point as the image of $X$ under the affinity obtained by first projecting $l$ to the line through $f_{l m}(X)$ and $f_{m n} f_{l m}(X)$ in the direction of $n$ and then projecting this line to $l$ in the direction of $A P$.

Since the intermediate line in this construction is parallel to $l$, this affinity belongs to $B(l)$, and since it sends 0 to $f_{n l} f_{m n} f_{l m}(X)$ it sends $X$ to $f_{n l} f_{m n} f_{l m}(X)+X$. But $f_{n l} f_{m n} f_{l m}$ sends 0 to 0 and $I$ to $A$, and is therefore $M_{I}^{A}$. It follows that $f_{m l} f_{l m}(X)=(A \cdot X)+X$. In particular, $f_{m l} f_{l m}(I)=(A \cdot I)+I=A+I$. But we also know that $f_{m l} f_{l m}$ belongs to $\mathfrak{N}_{0}(l)$. It must therefore be $M_{I}^{A+I}$. It follows that $(A \cdot X)+X$ $=(A+I) \cdot X$.

Corollary. $(-I) \cdot X+X=0$.

Proof. If $A=-I$ there is no parallel projection $f_{m l}$ but the same discussion applies to $f_{n l} f_{m n} f_{l m}$. If we define the function $f_{m l}$ by $f_{m l}(Y)=0$ for every $Y$ of $m$, then the argument given above shows that $0=((-I) \cdot X)+X$.

Corollary. $(A+B) \cdot X=(A \cdot X)+(B \cdot X)$.

Proof. If $B \neq 0$ the left distributive law yields $(A \cdot X)+(B \cdot X)$ $=B\left(\left(B^{-1} \cdot A \cdot X\right)+X\right)$ and the results just established apply.

We have just shown how each ordered pair of distinct points on a 
line gives rise to a field on that line. The various fields are related as follows.

Theorem 6. Let 0 and $I$ define field $F$ on line $l$, and let $0^{\prime}$ and $I^{\prime}$ define field $F^{\prime}$ on line $l^{\prime}$. Let $f$ be the affinity from $l$ to $l^{\prime}$ for which $f(0)=0^{\prime}$ and $f(I)=I^{\prime}$. Then $f$ is an isomorphism from $F$ to $F^{\prime}$.

Proof.

$$
\begin{aligned}
f(X \cdot Y) & =f\left(M_{I}^{X} M_{I}^{Y}(I)\right)=\left(f M_{I}^{X} f^{-1}\right)\left(f M_{I}^{Y} f^{-1}\right)(f(I))=M_{I^{\prime}}^{f(X)} M_{I^{\prime}}^{f(Y)}\left(I^{\prime}\right) \\
& =f(X) \cdot{ }^{\prime} f(Y), f(X+Y)=f\left(B_{0}^{X} B_{0}^{Y}(0)\right) \\
& =\left(f B_{0}^{X} f^{-1}\right)\left(f B_{0}^{Y} f^{-1}\right)(f(0))=B_{0^{\prime}}^{f(X)} B_{0^{\prime}}^{f(Y)}\left(0^{\prime}\right)=f(X)+^{\prime} f(Y) .
\end{aligned}
$$

The following theorem shows how a variant of Pappus' Theorem, expressed in terms of parallel projections, is related to the commutativity of the field.

THEOREM 7. The field is commutative if and only if, for every pair $l, l^{\prime}$ of intersecting lines and for every triple $f, g, h$ of parallel projections from $l$ to $l^{\prime}$ we have $\mathrm{fg}^{-1} h=h g^{-1}$.

Proof. Let $l$ and $l^{\prime}$ intersect at 0 and let $I$ be any other point of $l$. Multiplication is commutative if and only if, for every $X$ and $Y$ or $l$ other than 0 , we have $M_{I}^{X}(Y)=M_{I}^{Y}(X)$ or $M_{I}^{X} M_{I}^{Y}(I)=M_{I}^{Y} M_{I}^{X}(I)$. But in any case we have $M_{I}^{X} M_{I}^{Y}(0)=M_{I}^{Y} M_{I}^{X}(0)$. Therefore, by Axiom IV, multiplication is commutative if and only if $M_{I}^{X} M_{I}^{Y}=M_{I}^{Y} M_{I}^{X}$, or if and only if $\mathrm{Tl}_{0}(l)$ is commutative. It is clear that $g^{-1} f$ and $g^{-1} h$ are, respectively, the elements $M_{I}^{\sigma^{-1} f(I)}$ and $M_{I}^{\sigma^{-1} h(I)}$ of $\mathfrak{M}_{0}(l)$. It is also clear from the proof of Theorem 2 that each element of $\mathfrak{T}_{0}(l)$ has a representation $g^{-1} x$, where $g$ is the given parallel projection, and $x$ is a suitably chosen parallel projection. Therefore, multiplication is commutative if and only if, for every $f, g, h$, we have $g^{-1} f g^{-1} h=g^{-1} h g^{-1} f$, or $f g^{-1} h=h g^{-1} f$.

Coordinates. In order to work with a single field we select some line $l^{*}$, chose two distinct points $0^{*}$ and $I^{*}$ on it, and denote the field determined as a result of these choices by $F^{*}$. If $l$ is any line, if 0 and $I$ are any distinct points of $l$, if $f$ is the affinity for which $f(0)=0^{*}$ and $f(I)=I^{*}$ then, for every $X$ of $l$, we say that $f(X)$ is the $0 I$ coordinate of $X$.

Theorem 8. Let $l$ and $l^{\prime}$ be lines, 0 and I distinct points of $l, 0^{\prime}$ and $I^{\prime}$ distinct points of $l^{\prime}, g$ a function from $l$ to $l^{\prime}$. Then $g$ is an affinity if and only if there are elements $a$ and $b$ of $F^{*}$, with $b \neq 0^{*}$, such that for 
every $X$ of $l$ the $0^{\prime} I^{\prime}$ coordinate $x^{\prime}$ of $g(X)$ is related to the $0 I$ coordinate $x$ of $X$ by $x^{\prime}=a+b x$.

Proof. Case (1). $l=l^{\prime}=l^{*}, 0=0^{\prime}=0^{*}, I=I^{\prime}=I^{*}$. If $a$ and $b$ are any elements of $F^{*}$ with $b \neq 0^{*}$ then $B_{0}^{a} M_{I}^{b}$ is an affinity from $l$ to $l^{\prime}$ and is the map $x \rightarrow a+b x$. Conversely, if $g$ is an affinity from $l$ to $l$ let $a=g(0)$ and $b=g(I)-g(0)$. Then $b \neq 0$ and $B_{0}^{a} M_{I}^{b}$ is an affinity. Since it agrees with $g$ at 0 and $I$ it must be $g$.

Case (2). The lines $l, l^{\prime}, l^{*}$ are not necessarily the same. Let $f$ and $f^{\prime}$ be the affinities which map $0, I$ and $0^{\prime}, I^{\prime}$, respectively, onto $0^{*}, I^{*}$. Then $f^{\prime} g f^{-1}$ is a function from $l^{*}$ to itself which is an affinity if and only if $g$ is. Solving for $g$ and using Case (1), we see that $g$ is an affinity if and only if, for every $X$ of $l$, we have $f^{\prime}(g(X))=a+b f(X)$.

Corollary. If $P$ and $Q$ are distinct points of $l$, then there are elements $a$ and $b$ of $F^{*}$, with $b \neq 0^{*}$, such that for every $X$ of $l$ its $0 I$ coordinate $x$ is related to its $P Q$ coordinate $x^{\prime}$ by $x^{\prime}=a+b x$.

It is now possible to introduce $F^{*} \times F^{*}$ as a coordinate system for the plane and to show that lines have linear equations as in Levi $[3$, pp. 32-37], [4, pp. 50-53]. The arguments given there refer only to the field of real numbers but are valid for an arbitrary skew field.

The converse question. Now let $F$ be an arbitrary field (not necessarily commutative), let $\propto$ be $F \times F$ and let $\mathscr{L}$ be the family of subsets of $a$ whose elements are the solution sets of the equations $a x+b y+c$ $=0$, where $a, b, c$ are elements of $F$ and where not both $a$ and $b$ are 0 . We sketch a proof that $a$ and $\mathscr{L}$ satisfy Axioms I-IV. It is shown in Artin [1, pp. 66-67] that $a$ and $\mathscr{L}$ satisfy Axioms I, II, III and that if $P$ and $Q$ are any distinct points of $a$ a point $X$ is on the line which contains them if and only if there is a $t$ in $F$ such that $X=P$ $+(Q-P) t$. Call the unique $t$ associated in this way with a point $X$ of $P Q$ the $P Q$ coordinate of $X$. Then it is easy to see that if $f$ is a parallel projection from $P Q$ to $P^{\prime} Q^{\prime}$ for which $f(P)=P^{\prime}$ and $f(Q)=Q^{\prime}$, then, for every $X$ of $l$, the $P Q$ coordinate of $X$ equals the $P^{\prime} Q^{\prime}$ coordinate of $f(X)$. It follows that if a finite composition $g$ of parallel projections assigns $P^{*}$ and $Q^{*}$, respectively, to the distinct points $P$ and $Q$ then, for every $X$ of $P Q$, the $P Q$ coordinate of $X$ equals the $P^{*} Q^{*}$ coordinate of $g(X)$. This shows that an affinity is determined by two of its assignments and verifies Axiom IV.

Projective planes. We show how the foregoing treatment of the affine plane can be used to develop the projective plane. We suppose 
that $Q^{+}$is a set and that $\mathscr{L}^{+}$is a set of subsets of $a^{+}$. We all the elements of $a^{+}$points and the elements of $\mathfrak{L}^{+}$lines.

AxIom PI. If $A$ and $B$ are distinct points there is one and only one line which contains them.

AxIom PII. If $a$ and $b$ are lines there is a point on both.

Axiom PIII. There are four points no three of which are collinear.

It follows directly from these axioms that two distinct lines have exactly one common point, that every point is contained in at least three distinct lines, that every line contains at least three distinct points, and that all lines have the same cardinality. If $l$ and $l^{\prime}$ are lines and if $P$ is a point on neither then the perspectivity from $l$ to $l^{\prime}$ with center $P$ is the function whose value at each point $X$ of $l$ is the intersection of $P X$ and $l^{\prime}$. A projectivity is any finite composition of perspectivities.

For each line $l$ of $\mathfrak{L}^{+}$let $a(l)$ be the set of all points of $a^{+}$which are not on $l$. Each line of $\mathscr{L}$ other than $l$ has a unique representation $p \cup\{Q\}$, where $Q$ is on $l$ and where $p$ is a subset of $Q(l)$. We call each such subset $p$ an $l$-line and denote the set of all $l$-lines by $\mathcal{L}(l)$. We say that an $l$-line and the unique line of $\mathfrak{L}^{+}$which contains it are associated. It is easy to see that for every line $l$ of $\mathfrak{L}^{+}$the pair $Q(l)$, $\mathscr{L}(l)$ satisfy the Axioms I-III stated earlier for affine geometry. It is also easy to see that if the $l$-lines $p$ and $q$ are associated, respectively, with the lines $p \cup\{P\}$ and $q \cup\{Q\}$, then a function from $p$ to $q$ is a parallel projection, relative to $Q(l), \mathcal{L}(l)$ if and only if it is the restriction to $p$ of a perspectivity from $p \cup\{P\}$ to $q \cup\{Q\}$ whose center is on $l$. More generally, a function defined on the $l$-line $p$ is an affinity relative to $Q(l), \mathfrak{L}(l)$ if and only if it is the restriction to $p$ of a projectivity which is defined on $p \cup\{P\}$ and which is the composition of perspectivities whose centers are on $l$.

Our final axiom is:

Axiom PIV. There is a line $l_{0}$ such that if $A, B, A^{\prime}, B^{\prime}$ are points which are not on $l_{0}$ and if $A \neq A^{\prime}, B \neq B^{\prime}$, then there is at most one projectivity $f$, which is the composition of perspectivities whose centers are on $l_{0}$, and for which $f(A)=A^{\prime}, f(B)=B^{\prime}$.

It is immediate that $Q\left(l_{0}\right), \mathfrak{L}\left(l_{0}\right)$ satisfy our Axioms I-IV.

THEOREM 9. $Q^{+}$is the set of points and $\mathfrak{L}^{+}$is the set of lines of the projective plane obtained by adjoining $l_{0}$ as line at infinity to the affine plane $a\left(l_{0}\right)$.

Proof. Let $F$ be the field of the affine geometry $Q\left(l_{0}\right), \mathcal{L}\left(l_{0}\right)$ and let $Q\left(l_{0}\right) \rightarrow F \times F$ be an affine coordinate system for $Q\left(l_{0}\right)$. If $(u, v)$ are the affine coordinates of a point $X$ of $Q\left(b_{0}\right)$, assign to $X$ the equiva- 
lence class of triples $(u r, v r, r), r \in F, r \neq 0$. If $X$ is on $l_{0}$, let $a x+b y+c$ $=0$ be an equation of an $l$-line $p$ such that $p \cup\{X\}$ is a line of $\mathfrak{L}^{+}$, and assign to $X$ the equivalence class of triples $\left(-a^{-1} b r, r, 0\right), r \in F$, $r \neq 0$, if $a \neq 0$, and $(r, 0,0)$ if $a=0$. Then these assignments constitute a classical homogeneous coordinate system for $Q^{+}$relative to which the lines of $\mathfrak{L}^{+}$have equations $a x+b y+c z=0$, where $a, b, c$ are in $F$ and are not all 0 .

Corollary. If $\mathrm{Q}^{+}$and $\mathfrak{L}^{+}$satisfy Axioms PI-PIV relative to one line of $\mathfrak{L}^{+}$, they satisfy these Axioms relative to each line of $\mathfrak{L}^{+}$.

\section{REFERENCES}

1. E. Artin, Geometric algebra, Interscience, New York, 1957.

2. F. Bachmann, Aufbau der Geometrie aus dem Spiegelungsbegriff, Springer, Berlin, 1959.

3. H. Levi, Elements of geometry, Columbia University, New York, 1956.

4. - Foundations of geometry and trigonometry, Prentice-Hall, Englewood Cliffs, N. J., 1960.

5. G. Pickert, Projektive Ebenen, Springer, Berlin, 1955.

Hunter College, City University of New York 